\title{
Valores interpersonales y autoconcepto en estudiantes universitarios de la carrera de Ingeniería Ambiental y Administración de Empresas de una universidad estatal
}

\author{
Interpersonal values and self-concept in environmental engineering and business \\ administration students of a public state university \\ Rubén Sánchez P. ${ }^{1}$ y María Matalinares $C$. \\ Universidad Nacional Tecnológica del Cono Sur UNTECS, Lima, Perú \\ (RECIBIDO 18/03/2014, ACEPTADO 09/06/2014)
}

\begin{abstract}
RESUMEN
La presente investigación tiene como objetivo relacionar los valores interpersonales y el autoconcepto. La investigación es descriptiva, correlacional, comparativa y de diseño no experimental. La muestra estuvo constituida por 217 estudiantes universitarios de ambos sexos del primer, tercer y quinto ciclo de Ingeniería Ambiental y Administración de Empresas de una universidad estatal, a quienes se aplicó el cuestionario de autoconcepto forma (AF5), de Fernando García y Gonzalo Musitu, validado por Sandoval (2009) y Portilla (2011), y el cuestionario de valores interpersonales (SIV), de Leonard V. Gordon, adaptado por Higueras (1972) y Pérez (1987). Los resultados mostraron que existe correlación entre los valores interpersonales de soporte y conformidad con el autoconcepto general. En un análisis específico se observa que ningún valor interpersonal se relaciona con el autoconcepto familiar. Además se halló diferencias significativas en el autoconcepto físico y emocional, así como en el valor interpersonal de liderazgo. Por otro lado, no se observan diferencias estadísticas en los valores interpersonales y en el autoconcepto según la carrera profesional.
\end{abstract}

Palabras clave: valores interpersonales, autoconcepto, estudiantes universitarios.

\section{ABSTRACT}

The present research study aims to relate interpersonal values and self-concept. It is descriptive, correlational, comparative and with a non-experimental design. The sample consisted of 217 public state university students, both male and female, in their first, third and fifth cycles of Environmental Engineering and Business Administration careers. Fernando García and Gonzalo Musitu Self-Concept Questionnaire AF5 Form, validated by Sandoval (2009) and Portilla (2011), and Leonard V. Gordon Interpersonal Values Questionnaire (SIV), adapted by Higueras (1972) and Perez (1987), were the instruments applied. Results showed that there exist correlations between Support and Conformity Values and General

1 Docente de la Universidad Nacional Tecnológica del Cono Sur, UNTECS. Email: maria_luisa93@hotmail.com 
VALORES INTERPERSONALES Y AUTOCONCEPTO EN ESTUDIANTES UNIVERSITARIOS DE LA CARRERA DE INGENIERIA AMBIENTAL...

Self-Concept. In a more specific analysis, no relationship between any Interpersonal Value and Family Self-Concept was found. Furthermore, significant differences between Physical and Emotional Self-Concept and Leadership Interpersonal Value were found. On the other hand, there were not statistically significant differences between Interpersonal Values and Self-Concept concerning their professional careers.

Keywords: Interpersonal Values, Self-Concept, University Students

\section{INTRODUCCIÓN}

Los valores son parte importante en la formación, en la personalidad y la conducta de las personas que interactúan con otros dentro de un grupo social. En el caso concreto de los estudiantes universitarios, los valores además están vinculados al desarrollo de las competencias profesionales; no solo se les exige la adquisición de los conocimientos propios de la especialidad que han elegido, sino también que demuestren ser personas con valores personales e interpersonales que lo hagan un profesional competente (Campos, 2009, p. 79). Como sostienen Restrepo et al. (2009, p. 126 - 127), "la psicología en su intento de entender el fenómeno social se siente obligado a estudiar el sistema de valores que dirige el actuar humano". En la psicología los valores se han asumido como motivaciones que nos impulsan a actuar y en relación directa con la conducta (García, 2005, p. 2 - 3).Y, como afirma Molero (2003), "desde el punto de vista psicológico el estudio de los valores serviría para conocer las prioridades que orientan a las personas a la hora de adoptar decisiones y expresar unas determinadas actitudes" (p. 215). Para Rokeach (1968) los valores son guías del comportamiento y para Schwartz (1992) son las bases motivacionales de la acción (Álvaro et al. 2012, p. 101). Gordon (2003) afirma que "los valores pueden constituir un medio para determinar lo que los sujetos hacen y cómo lo hacen; muchas de sus decisiones inmediatas, así como sus planes a largo plazo están influidas, consciente o inconscientemente, por el sistema de valores que adopten" (Restrepo et al 2009, p.128).

En el Perú, investigadores como Berninzón (1996) examinó los valores interpersonales en alumnos de Psicología de la Universidad de San Martín de Porres. Fierro (1996) investigó la relación entre los valores interpersonales y el rendimiento académico en alumnos de Ingeniería Industrial y de Sistemas de la UNI. Quesquen (1997) investigó los valores interpersonales prevalentes en los estudiantes del I ciclo de las facultades de Ingeniería, Medicina, Educación y Derecho en la Universidad Nacional Mayor de San Marcos. Aguilar (2002) llevó a cabo un estudio comparativo de los valores interpersonales en alumnos con alta y baja autoestima de los tres primeros ciclos de la Escuela de Psicología de la Universidad Señor de Sipán, en la ciudad de Chiclayo. Carmona y León (2005) realizaron un análisis correlacional entre las inteligencias múltiples interpersonal e intrapersonal y los valores interpersonales, en estudiantes del primer ciclo de la Escuela de Psicología de la Universidad César Vallejo de Trujillo. Reyes (2003) llevó a cabo una 
investigación acerca de la relación entre el rendimiento académico, la ansiedad ante los exámenes, los rasgos de personalidad, el autoconcepto y la asertividad en estudiantes del primer año de Psicología de la UNMSM.

Ahora bien, el autoconcepto para Shavelson, Hubner y Stanton (1976) es "la percepción que el individuo tiene de sí mismo, la cual se basa directamente en sus experiencias en relación con los demás y en las atribuciones que él mismo hace de su propia conducta" (García y Musitu, 2001, p. 6). García y Musitu (2001) conciben el autoconcepto como un constructo que representa la concepción que el individuo tiene de sí mismo como ser físico, social y espiritual. De acuerdo a Pérez y Navarro (2011), el autoconcepto forma parte de la personalidad e identidad, y es la forma que tenemos de vernos en función de nuestra propia información interna (cogniciones) como de la externa que los demás reflejan sobre nosotros (p. 139). Quistgaard (1996) investigó los niveles de autoconcepto y conducta tipo A y B en estudiantes universitarios de Lima Metropolitana utilizando la escala de autoconcepto de Tennesse y el cuestionario de conducta tipo A - B de Eysenck y Fulker. Hernández (1998) realizó un estudio acerca de la relación del liderazgo y el autoconcepto en un grupo de estudiantes universitarios. Sandoval (2009) investigó el autoconcepto y los factores de protección asociados al consumo de alcohol y tabaco en universitarios de diferentes universidades de Lima. No obstante, el estudio sobre los valores interpersonales y el autoconcepto en el Perú apenas se ha iniciado.

\section{Hipótesis}

\section{Hipótesis general}

Los valores interpersonales de los estudiantes de la carrera de Ingeniería Ambiental y Administración de Empresas de una universidad estatal están relacionados significativamente con el autoconcepto.

\section{Hipótesis específicas}

1. Existen diferentes niveles en los valores interpersonales que presentan los estudiantes.

2. Existen diferentes niveles en el autoconcepto que presentan los estudiantes.

3. Existe diferencias significativas en los valores interpersonales de los estudiantes según el sexo.

4. Existe diferencias significativas en el autoconcepto de los estudiantes según el sexo.

5. Existe diferencias significativas en los valores interpersonales de los estudiantes según el ciclo académico y la carrera que cursan.

6. Existe diferencias en el autoconcepto de los estudiantes según el ciclo académico y la carrera que cursan. 


\section{MÉTODO}

\section{Tipo y diseño de investigación}

En concordancia con las hipótesis formuladas se empleó en un primer momento el método descriptivo con un diseño correlacional para relacionar las dos variables, y luego el diseño descriptivo comparativo para comparar los resultados de los estudiantes según el sexo, el ciclo académico y la carrera que cursan.

\section{Sujetos}

La muestra estuvo constituida por 217 estudiantes universitarios de ambos sexos del primer, tercer y quinto ciclo de Ingeniería Ambiental y Administración de Empresas de una universidad estatal, cuyas edades fluctuaban entre los 16 y 26 años.

\section{Técnicas e instrumentos}

Para el presente estudio se utilizó la técnica psicométrica y de observación indirecta.

Los instrumentos utilizados fueron:

\section{Cuestionario de autoconcepto forma 5 (AF5)}

Propuesto por Fernando García y Gonzalo Musitu (2001). Se aplica a adolescentes y adultos en forma individual o colectiva y su administración tiene una duración aproximadamente de 10 a 15 minutos. El cuestionario consta de 30 afirmaciones y evalúa 5 dimensiones del autoconcepto: académico, social, emocional, familiar y físico. Cada dimensión se evalúa con 6 ítems. La calificación de cada dimensión se obtiene asignando 1, 2 o 3 según la respuesta. La suma de los puntajes de las cinco dimensiones corresponde al autoconcepto general. Las dimensiones son: autopercepción académico-laboral, autoconcepto social, autoconcepto emocional, autoconcepto familiar y autoconcepto físico. Portilla (2011) realizó un análisis psicométrico para hallar la validez y confiabilidad del cuestionario AF5 en el Perú. Estableció la validez de contenido a través del criterio de jueces, los resultados indican que los ítems que componen el cuestionario AF5 superaron el valor mínimo de 0.80, por lo que se puede afirmar que este cuestionario posee una adecuada validez de contenido. En el análisis de confiabilidad mediante la consistencia interna, dio como resultado un coeficiente alfa de Cronbach de 0.872 , que indica que las puntuaciones que se obtienen con este cuestionario son confiables. Se observa también que las correlaciones ítem - test fluctúan entre 0.204 y 0.634 , sobrepasando el criterio de 0.20 , lo cual permite afirmar que los ítems del cuestionario son consistentes. Para la presente investigación el análisis de confiabilidad mediante el coeficiente de consistencia interna alfa de Cronbach fue de 0.755 , lo que indica la confiabilidad del cuestionario. Asimismo, de las dimensiones fueron los siguientes: autoconcepto académico - laboral, 0.824; autoconcepto social, 0.823; autoconcepto emocional, 0.844; autoconcepto familiar, 0.793 y autoconcepto físico, 0.641. En el análisis que se hizo, la consistencia interna más alta corresponde a la dimensión del autoconcepto emocional (0.844) y la más baja a la dimensión del autoconcepto físico (0.641). 


\section{Cuestionario de valores interpersonales (SIV)}

Se utilizó el cuestionario de valores interpersonales (SIV), de Leonard V. Gordon. La prueba fue adaptada en el Perú por Leonardo Higueras (1972) y Walter Pérez (1987). Mide los valores que se manifiestan en las relaciones con los demás. Estos valores tienen importancia en el ajuste personal, familiar, social y profesional de las personas. Se aplica a sujetos a partir de los 14 años de edad. Su aplicación puede ser individual o colectiva y tiene un tiempo de 15 minutos. El cuestionario contiene 90 enunciados agrupados de 3 en 3, constituyendo 30 series de 3 enunciados. Lo que se pide es leer los tres enunciados de cada serie y elegir el que es más importante y son los siguientes: estímulo, conformidad, reconocimiento, independencia, benevolencia y liderazgo. Para el presente estudio se realizó el análisis de confiabilidad del cuestionario y se encontró para las diferentes dimensiones los siguientes coeficientes alfa de Cronbach: independencia 0.682; soporte 0.566 ; benevolencia 0.768 ; conformidad 0.759 ; liderazgo 0.681 y reconocimiento 0.595. Los baremos fueron elaborados por el Dr. Leonardo Higueras (1972) a base de una muestra constituida por escolares y universitarios. La validez se realizó a través de la intercorrelación de factores aplicando el coeficiente de Pearson. Encontró que todas las correlaciones obtenidas tienen valores más altos que la r teórica, por lo que comprobó que el instrumento tiene validez. Su validez fue analizada y confirmada, elaborándose nuevos baremos por Walter Pérez (1987) con muestras de trabajadores y profesionales de educación secundaria y superior (Navarro, 2009). El SIV fue elaborado mediante la técnica del análisis factorial y, en este sentido, podemos señalar que las escalas del SIV tienen validez factorial.

\section{Procedimientos}

La recolección de la información se efectuó de manera colectiva, siguiendo las normas establecidas en los respectivos manuales. Las respuestas fueron calificadas en forma manual y se hizo una base de datos que fue procesada mediante el paquete estadístico SPPS versión 18.

\section{RESULTADOS}

\section{Análisis de los resultados descriptivos}

Tabla 1. Distribución de la dimensión independencia del cuestionario de valores interpersonales (SIV) en estudiantes de Ingeniería Ambiental y Administración $(\mathrm{N}=217)$

\begin{tabular}{ccccc}
\hline Dimensión & Nivel & Frecuencia (f) & Porcentaje (\%) \\
\hline \multirow{3}{*}{ Independencia } & Bajo & 93 & 42.9 \\
\cline { 2 - 5 } & Medio & 86 & 39.6 \\
\cline { 2 - 5 } & & Alto & 38 & 17.5 \\
\cline { 2 - 5 } & Total & & 217 & 100.0 \\
\hline
\end{tabular}

Se aprecia en la tabla 1 que en el valor interpersonal independencia, los estudiantes presentan un nivel predominantemente bajo (42.9\%), seguido de un nivel medio (39.6\%). 
VALORES INTERPERSONALES Y AUTOCONCEPTO EN ESTUDIANTES UNIVERSITARIOS DE LA CARRERA DE INGENIERIA AMBIENTAL...

Tabla 2. Distribución de la dimensión soporte del cuestionario de valores interpersonales (SIV) en estudiantes de Ingeniería Ambiental y Administración $(\mathrm{N}=217)$

\begin{tabular}{ccccc}
\hline Dimensión & Nivel & Frecuencia (f) & Porcentaje (\%) \\
\hline \multirow{3}{*}{ Soporte } & Bajo & 36 & 16.6 \\
\cline { 2 - 5 } & Medio & 92 & 42.4 \\
\cline { 2 - 5 } & & Alto & 89 & 41.0 \\
\hline & Total & & 217 & 100.0 \\
\hline
\end{tabular}

Como se puede observar en la tabla 2 el valor interpersonal soporte se distribuye predominantemente en un nivel medio (42.4\%), seguido de un nivel alto (41.0\%).

Tabla 3. Distribución de la dimensión benevolencia del cuestionario de valores interpersonales (SIV) en estudiantes de Ingeniería Ambiental y Administración $(\mathrm{N}=217)$

\begin{tabular}{ccccc}
\hline Dimensión & Nivel & Frecuencia (f) & Porcentaje (\%) \\
\hline \multirow{3}{*}{ Benevolencia } & Bajo & 95 & 43.8 \\
\cline { 2 - 5 } & & Medio & 73 & 33.6 \\
\cline { 2 - 5 } & & Alto & 49 & 22.6 \\
\hline & Total & & 217 & 100.0 \\
\hline
\end{tabular}

Se observa en la tabla 3 que en el valor interpersonal benevolencia, los estudiantes presentan un nivel predominantemente bajo (43.8\%), seguido de un nivel medio $(33.6 \%)$.

Tabla 4. Distribución de la dimensión conformidad del cuestionario de valores interpersonales (SIV) en estudiantes de Ingeniería Ambiental y Administración $(\mathrm{N}=217)$

\begin{tabular}{ccccc}
\hline Dimensión & Nivel & Frecuencia (f) & Porcentaje (\%) \\
\hline \multirow{3}{*}{ Conformidad } & Bajo & 42 & 19.4 \\
\cline { 2 - 5 } & & Medio & 80 & 36.9 \\
\cline { 2 - 5 } & & Alto & 95 & 43.8 \\
\hline & Total & & 217 & 100.0 \\
\hline
\end{tabular}

De acuerdo a la tabla 4 el valor interpersonal conformidad presenta mayoritariamente un nivel alto (43.8\%), seguido de un nivel medio (36.9\%).

Tabla 5. Distribución de la dimensión liderazgo del cuestionario de valores interpersonales (SIV) en estudiantes de Ingeniería Ambiental y Administración ( $\mathrm{N}=217)$

\begin{tabular}{ccccc}
\hline \multirow{2}{*}{ Dimensión } & Nivel & Frecuencia (f) & Porcentaje (\%) \\
\hline \multirow{3}{*}{ Liderazgo } & Bajo & 57 & 26.3 \\
\cline { 2 - 5 } & Medio & 75 & 34.6 \\
\cline { 2 - 5 } & & Alto & 85 & 39.2 \\
\hline & Total & & 217 & 100.0 \\
\hline
\end{tabular}

Se aprecia en la tabla 5 que el valor interpersonal liderazgo, presenta mayoritariamente un nivel alto (39.2\%), seguido de un nivel medio (34.6\%). 
Tabla 6. Distribución de la dimensión reconocimiento del cuestionario de valores interpersonales (SIV) en estudiantes de Ingeniería Ambiental y Administración $(\mathrm{N}=217)$

\begin{tabular}{ccccc}
\hline \multirow{2}{*}{ Dimensión } & Nivel & Frecuencia (f) & Porcentaje (\%) \\
\hline \multirow{3}{*}{ Reconocimiento } & Bajo & 51 & 23.5 \\
\cline { 2 - 5 } & Medio & 61 & 28.1 \\
\cline { 2 - 5 } & & Alto & 105 & 48.4 \\
\hline & Total & & 217 & 100.0 \\
\hline
\end{tabular}

En la tabla 6, el valor interpersonal reconocimiento presenta mayoritariamente un nivel alto $(48.4 \%)$, seguido de un nivel medio $(28.1 \%)$.

Tabla 7. Distribución de la variable autoconcepto en estudiantes de Ingeniería Ambiental y Administración $(\mathrm{N}=217)$

\begin{tabular}{ccccc}
\hline \multirow{2}{*}{ Variable } & Nivel & Frecuencia (f) & Porcentaje (\%) \\
\hline \multirow{3}{*}{ Autoconcepto } & Bajo & 54 & 24.9 \\
\cline { 2 - 5 } & Medio & 125 & 57.6 \\
\cline { 2 - 5 } & Alto & 38 & 17.5 \\
\hline & Total & & 217 & 100.0 \\
\hline
\end{tabular}

Según la tabla 7 se aprecia que el autoconcepto presenta en mayor proporción un nivel medio (57.6\%), seguido de un nivel bajo (24.9\%).

Tabla 8. Distribución de la dimensión académico del autoconcepto en estudiantes de Ingeniería Ambiental y Administración $(\mathrm{N}=217)$

\begin{tabular}{ccccc}
\hline \multirow{2}{*}{ Dimensión } & Nivel & Frecuencia (f) & Porcentaje (\%) \\
\hline \multirow{3}{*}{ Académico } & Bajo & 81 & 37.3 \\
\cline { 2 - 5 } & Medio & 82 & 37.8 \\
\cline { 2 - 5 } & Alto & 54 & 24.9 \\
\hline & Total & & 217 & 100.0 \\
\hline
\end{tabular}

Se observa en la tabla 8 que la dimensión académico presenta predominantemente un nivel medio (37.8\%), seguido de un nivel bajo (37.3\%).

Tabla 9. Distribución de la dimensión social del autoconcepto en estudiantes de Ingeniería Ambiental y Administración ( $\mathrm{N}=217)$

\begin{tabular}{ccccc}
\hline \multirow{3}{*}{ Dimensión } & Nivel & Frecuencia (f) & Porcentaje (\%) \\
\hline \multirow{3}{*}{ Social } & Bajo & 71 & 32.7 \\
\cline { 2 - 5 } & & Medio & 141 & 65.0 \\
\cline { 2 - 5 } & & Alto & 5 & 2.3 \\
\hline & Total & & 217 & 100.0 \\
\hline
\end{tabular}

Se tiene en la tabla 9 que la dimensión social presenta predominantemente un nivel medio $(65.0 \%)$, seguido de un nivel bajo $(32.7 \%)$. 
VALORES INTERPERSONALES Y AUTOCONCEPTO EN ESTUDIANTES UNIVERSITARIOS DE LA CARRERA DE INGENIERIA AMBIENTAL...

Tabla 10. Distribución de la dimensión emocional del autoconcepto en estudiantes de Ingeniería Ambiental y Administración $(\mathrm{N}=217)$

\begin{tabular}{ccccc}
\hline \multirow{2}{*}{ Dimensión } & Nivel & Frecuencia (f) & Porcentaje (\%) \\
\hline \multirow{3}{*}{ Emocional } & Bajo & 41 & 18.9 \\
\cline { 2 - 5 } & Medio & 120 & 55.3 \\
\cline { 2 - 5 } & Alto & 56 & 25.8 \\
\hline & Total & & 217 & 100.0 \\
\hline
\end{tabular}

Se aprecia en la tabla 10 que la dimensión emocional presenta en mayor proporción un nivel medio (55.3\%), seguido de un nivel bajo (25.8\%).

Tabla 11. Distribución de la dimensión familiar del autoconcepto en estudiantes de Ingeniería Ambiental y Administración (N=217)

\begin{tabular}{ccccc}
\hline \multirow{2}{*}{ Dimensión } & Nivel & Frecuencia (f) & Porcentaje (\%) \\
\hline \multirow{3}{*}{ Familiar } & Bajo & 38 & 17.5 \\
\cline { 2 - 5 } & Medio & 114 & 52.5 \\
\cline { 2 - 5 } & Tlto & 65 & 30.0 \\
\hline & Total & & 217 & 100.0 \\
\hline
\end{tabular}

Se observa en la tabla 11 que la dimensión familiar presenta mayoritariamente un nivel medio $(52.5 \%)$, seguido de un nivel alto (30.0\%).

Tabla 12. Distribución de la dimensión físico del autoconcepto en estudiantes de Ingeniería Ambiental y Administración $(\mathrm{N}=217)$

\begin{tabular}{ccccc}
\hline \multirow{2}{*}{ Dimensión } & Nivel & Frecuencia (f) & Porcentaje (\%) \\
\hline \multirow{3}{*}{ Físico } & Bajo & 61 & 28.1 \\
\cline { 2 - 5 } & & Medio & 95 & 43.8 \\
\cline { 2 - 5 } & & Alto & 61 & 28.1 \\
\hline & Total & & 217 & 100.0 \\
\hline
\end{tabular}

De acuerdo a la tabla 12, la dimensión físico presenta predominantemente un nivel medio (43.8\%), seguido en similar proporción por los niveles bajo y alto (28.1\%).

Tabla 13. Estadísticos descriptivos y prueba de normalidad de los valores interpersonales en estudiantes de Ingeniería Ambiental y Administración $(\mathrm{N}=217)$

\begin{tabular}{|c|c|c|c|c|c|}
\hline \multirow{2}{*}{ Dimensiones } & \multirow{2}{*}{$\mathrm{N}$} & \multicolumn{2}{|c|}{ Parámetros normales } & \multirow{2}{*}{$\begin{array}{c}\mathrm{Z} \text { de } \\
\text { Kolmogorov- } \\
\text { Smirnov }\end{array}$} & \multirow{2}{*}{$\begin{array}{l}\text { Sig. asintót } \\
\text { (bilateral) }\end{array}$} \\
\hline & & Media & Desviación típica & & \\
\hline Independencia & 217 & 16.38 & 5.09 & 1.049 & 0.221 \\
\hline Soporte & 217 & 13.59 & 4.23 & 0.981 & 0.291 \\
\hline Benevolencia & 217 & 17.65 & 5.33 & 1.302 & 0.067 \\
\hline
\end{tabular}


Rubén Sánchez P. y María Matalinares C.

$\begin{array}{cccccc}\text { Conformidad } & 217 & 16.69 & 5.29 & 1.039 & 0.231 \\ \text { Liderazgo } & 217 & 16.11 & 5.19 & 0.978 & 0.294 \\ \text { Reconocimiento } & 217 & 9.58 & 3.89 & 1.111 & 0.169\end{array}$

En la tabla 14 se presentan los estadísticos descriptivos de las valores interpersonales. Así, el valor independencia presenta una media de 16.38 y dispersión de 5.09; el valor soporte tiene un promedio de 13.59 y desviación de 4.23; el valor benevolencia posee una media de 17.65 y dispersión de 5.33; el valor conformidad posee una media de 16.69 y dispersión de 5.29; el valor liderazgo obtuvo un promedio de 16.69 y desviación de 5.29; y el valor reconocimiento alcanzó una media de 9.58 y desviación de 3.89. En la misma tabla 14 se aplica la prueba de normalidad de Kolmogorov-Smirnov encontrándose en todos los valores interpersonales un p-valor superior al 0.05. Por tanto, la muestra en los valores interpersonales posee una distribución normal, por lo cual se hará uso de las pruebas paramétricas en el análisis inferencial.

Tabla 14. Estadísticos descriptivos y prueba de normalidad de la variable autoconcepto y sus dimensiones en estudiantes de Ingeniería Ambiental y Administración $(\mathrm{N}=217)$

\begin{tabular}{|c|c|c|c|c|c|}
\hline \multirow{2}{*}{$\begin{array}{l}\text { Variables y } \\
\text { dimensiones }\end{array}$} & \multirow{2}{*}{$\mathrm{N}$} & \multicolumn{2}{|c|}{ Parámetros normales } & \multirow{2}{*}{$\begin{array}{c}\mathrm{Z} \text { de } \\
\text { Kolmogorov- } \\
\text { Smirnov }\end{array}$} & \multirow{2}{*}{$\begin{array}{l}\text { Sig. asintót } \\
\text { (bilateral) }\end{array}$} \\
\hline & & Media & Desviación típica & & \\
\hline Autoconcepto & 217 & 107.60 & 10.63 & 0.706 & 0.701 \\
\hline $\begin{array}{l}\text { Dimensión } \\
\text { académico }\end{array}$ & 217 & 21.21 & 3.10 & 1.725 & 0.005 \\
\hline Dimensión social & 217 & 20.96 & 2.23 & 1.964 & 0.001 \\
\hline $\begin{array}{l}\text { Dimensión } \\
\text { emocional }\end{array}$ & 217 & 19.65 & 3.85 & 1.571 & 0.014 \\
\hline $\begin{array}{l}\text { Dimensión } \\
\text { familiar }\end{array}$ & 217 & 25.10 & 3.49 & 2.415 & 0.000 \\
\hline Dimensión físico & 217 & 20.67 & 3.71 & 0.961 & 0.314 \\
\hline
\end{tabular}

De acuerdo a la tabla 14, se presentan los estadísticos descriptivos de la variable autoconcepto y sus dimensiones. La variable autoconcepto presenta una media de 107.60 y desviación de 10.63. La dimensión académico se obtiene la media de 21.21 y dispersión de 3.10; la dimensión social alcanza un promedio de 20.96 y dispersión de 2.23; la dimensión emocional posee una media de 19.65 y desviación de 3.85; la dimensión familiar tiene un promedio de 25.10 y dispersión de 3.49; y la dimensión físico alcanza una media de 20.67 y dispersión de 3.71. 
Dentro de la misma tabla 14, se aplica la prueba de normalidad de KolmogorovSmirnov, donde la variable autoconcepto y dimensión físico obtienen un p-valor superior a 0.05 ; por ello, en ambos casos se aplicará las pruebas paramétricas para los análisis inferenciales. En contraste, las dimensiones académico, social, emocional y familiar obtuvieron un p-valor inferior al 0.05; en tal sentido, se aplicará, en dichos casos, las pruebas no paramétricas para los análisis inferenciales.

\section{Análisis de los resultados inferenciales}

Tabla 15. Correlaciones entre la variable autoconcepto y los valores interpersonales en estudiantes de Ingeniería Ambiental y Administración $(\mathrm{N}=217)$

\begin{tabular}{|c|c|c|}
\hline Valores interpersonales & & Autoconcepto \\
\hline \multirow{3}{*}{ Independencia } & R Pearson & .057 \\
\hline & Sig. (bilateral) & .403 \\
\hline & $\mathrm{N}$ & 217 \\
\hline \multirow{3}{*}{ Soporte } & R Pearson & $-.288(* *)$ \\
\hline & Sig. (bilateral) & .000 \\
\hline & $\mathrm{N}$ & 217 \\
\hline \multirow{3}{*}{ Benevolencia } & R Pearson & -.081 \\
\hline & Sig. (bilateral) & .236 \\
\hline & $\mathrm{N}$ & 217 \\
\hline \multirow{3}{*}{ Conformidad } & R Pearson & .121 \\
\hline & Sig. (bilateral) & .076 \\
\hline & $\mathrm{N}$ & 217 \\
\hline \multirow{3}{*}{ Liderazgo } & R Pearson & $.154(*)$ \\
\hline & Sig. (bilateral) & .024 \\
\hline & $\mathrm{N}$ & 217 \\
\hline \multirow{3}{*}{ Reconocimiento } & R Pearson & -.020 \\
\hline & Sig. (bilateral) & .769 \\
\hline & $\mathrm{N}$ & 217 \\
\hline
\end{tabular}

* La correlación es significativa al nivel 0.05 (bilateral).

** La correlación es significativa al nivel 0.01 (bilateral).

En la tabla 15, se presentan las correlaciones entre la variable autoconcepto y los valores interpersonales mediante el estadístico paramétrico de $\mathrm{R}$ de Pearson. En ellos, se puede identificar una correlación inversa, media y muy significativa $(\mathrm{p}<0.01)$ entre el autoconcepto y el valor soporte; así también, se obtuvo una correlación directa, media y significativa $(\mathrm{p}<0.05)$ entre el autoconcepto y el valor liderazgo. 
Tabla 16. Correlaciones entre las dimensiones de la variable autoconcepto y los valores interpersonales en estudiantes de Ingeniería Ambiental y Administración $(\mathrm{N}=217)$

\begin{tabular}{|c|c|c|c|c|c|}
\hline $\begin{array}{c}\text { Valores } \\
\text { interpersonales }\end{array}$ & & $\begin{array}{l}\text { Dimensión } \\
\text { académico }\end{array}$ & $\begin{array}{l}\text { Autoconcepto } \\
\text { Dimensión } \\
\text { social }\end{array}$ & $\begin{array}{l}\text { Dimensión } \\
\text { emocional }\end{array}$ & $\begin{array}{c}\text { Dimensión } \\
\text { familiar }\end{array}$ \\
\hline \multirow{3}{*}{ Independencia } & $\begin{array}{c}\text { Rho de } \\
\text { Spearman }\end{array}$ & .011 & -.029 & $.247(* *)$ & -.005 \\
\hline & Sig. (bilateral) & .869 & .674 & .000 & .937 \\
\hline & $\mathrm{N}$ & 217 & 217 & 217 & 217 \\
\hline \multirow{3}{*}{ Soporte } & $\begin{array}{c}\text { Rho de } \\
\text { Spearman }\end{array}$ & $-.287(* *)$ & -.127 & $-.146\left(^{*}\right)$ & -.118 \\
\hline & Sig. (bilateral) & .000 & .062 & .031 & .084 \\
\hline & $\mathrm{N}$ & 217 & 217 & 217 & 217 \\
\hline \multirow{3}{*}{ Benevolencia } & $\begin{array}{c}\text { Rho de } \\
\text { Spearman }\end{array}$ & -.052 & -.006 & $-.147(*)$ & .071 \\
\hline & Sig. (bilateral) & .447 & .932 & .030 & .297 \\
\hline & $\mathrm{N}$ & 217 & 217 & 217 & 217 \\
\hline \multirow{3}{*}{ Conformidad } & $\begin{array}{c}\text { Rho de } \\
\text { Spearman }\end{array}$ & $.156(*)$ & $.181(* *)$ & -.103 & .055 \\
\hline & Sig. (bilateral) & .022 & .007 & .130 & .423 \\
\hline & $\mathrm{N}$ & 217 & 217 & 217 & 217 \\
\hline \multirow{3}{*}{ Liderazgo } & $\begin{array}{c}\text { Rho de } \\
\text { Spearman }\end{array}$ & $.194(* *)$ & .014 & $.157\left(^{*}\right)$ & .071 \\
\hline & Sig. (bilateral) & .004 & .835 & .021 & .298 \\
\hline & $\mathrm{N}$ & 217 & 217 & 217 & 217 \\
\hline \multirow{3}{*}{ Reconocimiento } & $\begin{array}{c}\text { Rho de } \\
\text { Spearman }\end{array}$ & -.049 & -.029 & .012 & -.079 \\
\hline & Sig. (bilateral) & .473 & .673 & .857 & .249 \\
\hline & $\mathrm{N}$ & 217 & 217 & 217 & 217 \\
\hline
\end{tabular}

** La correlación es significativa al nivel 0.01 (bilateral).

* La correlación es significativa al nivel 0.05 (bilateral).

Se aprecia en la tabla 16 las correlaciones entre los valores interpersonales y las dimensiones académico, ssocial, emocional y familiar de la variable autoconcepto mediante la prueba no paramétrica Rho de Spearman. En la dimensión académico, se detectó una correlación inversa, media y muy significativa $(\mathrm{p}<0.01)$ con el valor soporte; además, una correlación directa, media y muy significativa $(\mathrm{p}<0.01)$ con los valores conformidad y liderazgo. Respecto a la dimensión social, 
se encontró una correlación directa, media y muy significativa $(p<0.01)$ con el valor conformidad. En relación a la dimensión emocional se halló una correlación directa, media y significativa $(\mathrm{p}<0.01)$ con el valor independencia; asimismo, una correlación inversa, media y significativa $(\mathrm{p}<0.05)$ con los valores soporte y benevolencia; $y$, una correlación directa, media y significativa $(p<0.05)$ con el valor liderazgo. Finalmente, en la dimensión familiar no se obtuvo correlaciones significativas.

Tabla 17. Correlaciones entre las dimensiones de la variable autoconcepto y los valores interpersonales en estudiantes de Ingeniería Ambiental y Administración $(\mathrm{N}=217)$

\begin{tabular}{|c|c|c|}
\hline Valores interpersonales & & Dimensión físico \\
\hline \multirow{3}{*}{ Independencia } & R Pearson & .005 \\
\hline & Sig. (bilateral) & .944 \\
\hline & $\mathrm{N}$ & 217 \\
\hline \multirow{3}{*}{ Soporte } & R Pearson & $-.241(* *)$ \\
\hline & Sig. (bilateral) & .000 \\
\hline & $\mathrm{N}$ & 217 \\
\hline \multirow{3}{*}{ Benevolencia } & R Pearson & -.087 \\
\hline & Sig. (bilateral) & .203 \\
\hline & $\mathrm{N}$ & 217 \\
\hline \multirow{3}{*}{ Conformidad } & R Pearson & $.162(*)$ \\
\hline & Sig. (bilateral) & .017 \\
\hline & $\mathrm{N}$ & 217 \\
\hline \multirow{3}{*}{ Liderazgo } & R Pearson & .084 \\
\hline & Sig. (bilateral) & .217 \\
\hline & $\mathrm{N}$ & 217 \\
\hline \multirow{3}{*}{ Reconocimiento } & R Pearson & .043 \\
\hline & Sig. (bilateral) & .533 \\
\hline & $\mathrm{N}$ & 217 \\
\hline
\end{tabular}

** La correlación es significativa al nivel 0.01 (bilateral).

* La correlación es significativa al nivel 0.05 (bilateral).

De acuerdo a la tabla 17 tenemos las correlaciones entre la dimensión físico del autoconcepto y los valores interpersonales mediante la prueba paramétrica de $\mathrm{R}$ de Pearson. Se aprecia una correlación inversa, media y muy significativa $(\mathrm{p}<0.01)$ entre la dimensión físico con el valor soporte; así también, una correlación directa, media y significativa $(\mathrm{p}<0.05)$ entre la dimensión físico con el valor conformidad. 
Tabla 18. Prueba de T de Student para muestras independientes para estimar si existen diferencias de la variable autoconcepto y su dimensión físico según sexo

\begin{tabular}{ccccccc}
\hline Variable y dimensiones & Grupo & $\mathrm{N}$ & Media & $\mathrm{t}$ & $\mathrm{gl}$ & $\begin{array}{c}\text { Sig. } \\
\text { (bilateral) }\end{array}$ \\
\hline \multirow{2}{*}{ Autoconcepto } & Masculino & 72 & 108.75 & & & \\
& Femenino & 145 & 107.03 & 1.124 & 215 & .262 \\
\hline \multirow{2}{*}{ Dimensión físico } & Masculino & 72 & 21.86 & & & \\
& Femenino & 145 & 20.08 & & & \\
\hline
\end{tabular}

** Diferencias significativas al nivel 0.01 (bilateral).

En la tabla 18 se aprecian las comparaciones según sexo de la variable autoconcepto y su dimensión físico mediante la prueba paramétrica T de Student para muestras independientes. Existen diferencias altamente significativas $(\mathrm{p}<0.01)$ de la dimensión físico a favor de los varones.

Tabla 19. Prueba no paramétrica U de Mann-Whitney para muestras independientes para estimar si existen diferencias de las dimensiones académico, social, emocional y familiar según sexo

\begin{tabular}{|c|c|c|c|c|c|c|}
\hline Dimensiones & Grupo & $\mathrm{N}$ & $\begin{array}{c}\text { Rango } \\
\text { promedio }\end{array}$ & $\begin{array}{c}\text { U de Mann- } \\
\text { Whitney }\end{array}$ & $\mathrm{Z}$ & $\begin{array}{c}\text { Sig. } \\
\text { (bilateral) }\end{array}$ \\
\hline \multirow{2}{*}{$\begin{array}{l}\text { Dimensión } \\
\text { académico }\end{array}$} & Masculino & 72 & 98.69 & \multirow{2}{*}{4478.000} & \multirow{2}{*}{-1.714} & \multirow{2}{*}{.087} \\
\hline & Femenino & 145 & 114.12 & & & \\
\hline \multirow{3}{*}{ Dimensión social } & Masculino & 72 & 98.67 & \multirow{3}{*}{4476.500} & \multirow{3}{*}{-1.728} & \multirow{3}{*}{.084} \\
\hline & & & & & & \\
\hline & Femenino & 145 & 114.13 & & & \\
\hline \multirow{2}{*}{$\begin{array}{l}\text { Dimensión } \\
\text { emocional }\end{array}$} & Masculino & 72 & 127.55 & \multirow{2}{*}{3884.500} & \multirow{2}{*}{-3.081} & \multirow{2}{*}{$.002(* *)$} \\
\hline & Femenino & 145 & 99.79 & & & \\
\hline \multirow{3}{*}{ Dimensión familiar } & Masculino & 72 & 106.73 & \multirow{3}{*}{5056.500} & \multirow{3}{*}{-.377} & \multirow{3}{*}{.706} \\
\hline & & & & & & \\
\hline & Femenino & 145 & 110.13 & & & \\
\hline
\end{tabular}

** Diferencias significativas al nivel 0.01 (bilateral).

En la tabla 19 se tienen las comparaciones de las dimensiones académico, social, emocional y familiar según sexo, utilizando la prueba no paramétrica de $\mathrm{U}$ de Mann-Whitney. Se detectó diferencias altamente significativas $(\mathrm{p}<0.01)$ en el autoconcepto emocional a favor de los varones. 
VALORES INTERPERSONALES Y AUTOCONCEPTO EN ESTUDIANTES UNIVERSITARIOS DE LA CARRERA DE INGENIERIA AMBIENTAL...

Tabla 20. Prueba de T de Student para muestras independientes para estimar si existen diferencias en los valores interpersonales según sexo

\begin{tabular}{|c|c|c|c|c|c|c|}
\hline Dimensiones & Grupo & $\mathrm{N}$ & Media & $\mathrm{t}$ & gl & Sig. (bilateral) \\
\hline \multirow{2}{*}{ Independencia } & Masculino & 72 & 17.19 & \multirow{2}{*}{1.662} & \multirow{2}{*}{215} & \multirow{2}{*}{.098} \\
\hline & Femenino & 145 & 15.98 & & & \\
\hline \multirow{2}{*}{ Soporte } & Masculino & 72 & 13.86 & \multirow{2}{*}{.677} & \multirow{2}{*}{215} & \multirow{2}{*}{.499} \\
\hline & Femenino & 145 & 13.45 & & & \\
\hline \multirow{2}{*}{ Benevolencia } & Masculino & 72 & 17.06 & \multirow{2}{*}{-1.159} & \multirow{2}{*}{215} & \multirow{2}{*}{.248} \\
\hline & Femenino & 145 & 17.94 & & & \\
\hline \multirow{2}{*}{ Conformidad } & Masculino & 72 & 17.51 & \multirow{2}{*}{1.628} & \multirow{2}{*}{215} & \multirow{2}{*}{.105} \\
\hline & Femenino & 145 & 16.28 & & & \\
\hline \multirow{2}{*}{ Liderazgo } & Masculino & 72 & 15.08 & \multirow{2}{*}{-2.069} & \multirow{2}{*}{215} & \multirow{2}{*}{$.040(*)$} \\
\hline & Femenino & 145 & 16.62 & & & \\
\hline \multirow{2}{*}{ Reconocimiento } & Masculino & 72 & 9.29 & \multirow{2}{*}{-.770} & \multirow{2}{*}{215} & \multirow{2}{*}{.442} \\
\hline & Femenino & 145 & 9.72 & & & \\
\hline
\end{tabular}

* Diferencias significativas al nivel 0.05 (bilateral).

En la tabla 20 se aplicó la prueba paramétrica T de Student para muestras independientes a fin de establecer las diferencias según sexo de los valores interpersonales. Se aprecian diferencias significativas $(\mathrm{p}<0.05)$ en el valor liderazgo a favor de las mujeres.

Tabla 21. Prueba de T de Student para muestras independientes para estimar si existen diferencias de la variable autoconcepto y su dimensión físico según carrera profesional

\begin{tabular}{|c|c|c|c|c|c|c|}
\hline Variable y dimensiones & Grupo & $\mathrm{N}$ & Media & $\mathrm{t}$ & gl & Sig. (bilateral) \\
\hline \multirow[b]{2}{*}{ Autoconcepto } & Administración & 104 & 108.31 & \multirow[b]{2}{*}{.941} & \multirow[b]{2}{*}{215} & \multirow[b]{2}{*}{.348} \\
\hline & $\begin{array}{l}\text { Ingeniería } \\
\text { Ambiental }\end{array}$ & 113 & 106.95 & & & \\
\hline \multirow[b]{2}{*}{ Dimensión físico } & Administración & 104 & 20.85 & \multirow[b]{2}{*}{.677} & \multirow[b]{2}{*}{215} & \multirow[b]{2}{*}{.499} \\
\hline & $\begin{array}{l}\text { Ingeniería } \\
\text { Ambiental }\end{array}$ & 113 & 20.50 & & & \\
\hline
\end{tabular}

* Diferencias significativas al nivel 0.05 (bilateral).

En relación con la carrera profesional, se observa en la tabla 21 las comparaciones de la variable autoconcepto y su dimensión físico a través de la prueba paramétrica T de Student. En estas no existen diferencias significativas. 
Tabla 22. Prueba no paramétrica U de Mann-Whitney para muestras independientes para estimar si existen diferencias en las dimensiones académico, social, emocional y familiar según la carrera profesional

\begin{tabular}{|c|c|c|c|c|c|c|}
\hline Dimensiones & Grupo & $\mathrm{N}$ & $\begin{array}{c}\text { Rango } \\
\text { promedio }\end{array}$ & $\begin{array}{c}\text { U de Mann- } \\
\text { Whitney }\end{array}$ & $\mathrm{Z}$ & $\begin{array}{c}\text { Sig. } \\
\text { (bilateral) }\end{array}$ \\
\hline \multirow{2}{*}{$\begin{array}{l}\text { Dimensión } \\
\text { académico }\end{array}$} & Administración & 104 & 115.46 & \multirow{2}{*}{5204.500} & \multirow{2}{*}{-1.462} & \multirow{2}{*}{.144} \\
\hline & $\begin{array}{l}\text { Ingeniería } \\
\text { Ambiental }\end{array}$ & 113 & 103.06 & & & \\
\hline \multirow{2}{*}{$\begin{array}{l}\text { Dimensión } \\
\text { social }\end{array}$} & Administración & 104 & 111.35 & \multirow[b]{2}{*}{5631.500} & \multirow[b]{2}{*}{-.536} & \multirow[b]{2}{*}{.592} \\
\hline & $\begin{array}{l}\text { Ingeniería } \\
\text { Ambiental }\end{array}$ & 113 & 106.84 & & & \\
\hline \multirow{2}{*}{$\begin{array}{l}\text { Dimensión } \\
\text { emocional }\end{array}$} & Administración & 104 & 106.33 & \multirow{2}{*}{5598.000} & \multirow{2}{*}{-.604} & \multirow{2}{*}{.546} \\
\hline & $\begin{array}{l}\text { Ingeniería } \\
\text { Ambiental }\end{array}$ & 113 & 111.46 & & & \\
\hline \multirow{2}{*}{$\begin{array}{l}\text { Dimensión } \\
\text { familiar }\end{array}$} & Administración & 104 & 112.17 & \multirow[b]{2}{*}{5546.000} & \multirow[b]{2}{*}{-.718} & \multirow[b]{2}{*}{.473} \\
\hline & $\begin{array}{l}\text { Ingeniería } \\
\text { Ambiental }\end{array}$ & 113 & 106.08 & & & \\
\hline
\end{tabular}

* Diferencias significativas al nivel 0.05 (bilateral).

Se tiene en la tabla 22 las comparaciones de las dimensiones académico, social, emocional y familiar, utilizando la prueba no paramétrica U de Mann-Whitney. Así, en estas no se logran obtener diferencias significativas según la carrera profesional.

Tabla 23. Prueba de $\mathrm{T}$ de Student para muestras independientes para estimar si existen diferencias en los valores interpersonales según la carrera profesional

\begin{tabular}{ccccccc}
\hline Dimensiones & Grupo & $\mathrm{N}$ & Media & $\mathrm{t}$ & $\mathrm{gl}$ & Sig. (bilateral) \\
\hline \multirow{2}{*}{ Independencia } & Administración & 104 & 16.00 & & & \\
& $\begin{array}{c}\text { Ingeniería } \\
\text { Ambiental }\end{array}$ & 113 & 16.73 & -1.062 & 215 & .289 \\
\hline Soporte & Administración & 104 & 13.77 & & & \\
& $\begin{array}{c}\text { Ingeniería } \\
\text { Ambiental }\end{array}$ & 113 & 13.42 & & & .540 \\
\hline
\end{tabular}


VALORES INTERPERSONALES Y AUTOCONCEPTO EN ESTUDIANTES UNIVERSITARIOS DE LA CARRERA DE INGENIERIAA AMBIENTAL...

\begin{tabular}{|c|c|c|c|c|c|c|}
\hline \multirow{3}{*}{ Benevolencia } & Administración & 104 & 16.93 & \multirow{3}{*}{-1.914} & \multirow{3}{*}{215} & \multirow{3}{*}{.057} \\
\hline & \multirow[b]{2}{*}{$\begin{array}{l}\text { Ingeniería } \\
\text { Ambiental }\end{array}$} & & & & & \\
\hline & & 113 & 18.31 & & & \\
\hline \multirow{3}{*}{ Conformidad } & Administración & 104 & 16.73 & \multirow{3}{*}{.118} & \multirow{3}{*}{215} & \multirow{3}{*}{.907} \\
\hline & \multirow[b]{2}{*}{$\begin{array}{l}\text { Ingeniería } \\
\text { Ambiental }\end{array}$} & & & & & \\
\hline & & 113 & 16.65 & & & \\
\hline \multirow{3}{*}{ Liderazgo } & Administración & 104 & 16.71 & \multirow{3}{*}{1.642} & \multirow{3}{*}{215} & \multirow{3}{*}{.102} \\
\hline & \multirow{2}{*}{$\begin{array}{l}\text { Ingeniería } \\
\text { Ambiental }\end{array}$} & & & & & \\
\hline & & 113 & 15.56 & & & \\
\hline \multirow{3}{*}{ Reconocimiento } & Administración & 104 & 9.85 & \multirow{3}{*}{.964} & \multirow{3}{*}{215} & \multirow{3}{*}{.336} \\
\hline & & & & & & \\
\hline & $\begin{array}{l}\text { Ingeniería } \\
\text { Ambiental }\end{array}$ & 113 & 9.34 & & & \\
\hline
\end{tabular}

* Diferencias significativas al nivel 0.05 (bilateral).

De acuerdo a la tabla 23 se realizaron las comparaciones en los valores interpersonales a través de la prueba paramétrica $\mathrm{T}$ de Student para muestras independientes. Se aprecia que no hay diferencias estadísticamente significativas según la carrera profesional.

Tabla 24. Prueba de Anova de un factor para estimar si existen diferencias de la variable autoconcepto y su dimensión físico según ciclo de estudios

\begin{tabular}{ccccccc}
\hline $\begin{array}{c}\text { Variable y } \\
\text { dimensiones }\end{array}$ & Grupo & $\begin{array}{c}\text { Suma de } \\
\text { cuadrados }\end{array}$ & gl & $\begin{array}{c}\text { Media } \\
\text { cuadrática }\end{array}$ & F & Sig. \\
\hline Anter-grupos & 15.318 & 2 & & & \\
& Tntra-grupos & 24408.802 & 214 & 7.659 & .067 & .935 \\
& Total & 24424.120 & 216 & & & \\
\hline & Inter-grupos & 9.706 & 2 & & & \\
Dimensión físico & Intra-grupos & 2962.404 & 214 & 4.853 & .351 & .705 \\
& Total & 2972.111 & 216 & & & \\
\hline
\end{tabular}

* Diferencias significativas al nivel 0.05 (bilateral).

Se observa en la tabla 24 las comparaciones según ciclo de estudios en la variable autoconcepto y su dimensión físico mediante la prueba paramétrica Anova de un factor. En ambos casos, no se detectaron diferencias significativas. 
Tabla 25. Prueba no paramétrica Kruskal-Wallis para estimar si existen diferencias de las dimensiones académico, social, emocional y familiar según ciclo de estudios

\begin{tabular}{|c|c|c|c|c|c|c|}
\hline Dimensiones & Grupos & $\mathrm{N}$ & Rango promedio & $\begin{array}{c}\text { Chi- } \\
\text { cuadrado }\end{array}$ & $\mathrm{gl}$ & $\begin{array}{c}\text { Sig. } \\
\text { (bilateral) }\end{array}$ \\
\hline \multirow{3}{*}{$\begin{array}{l}\text { Dimensión } \\
\text { académico }\end{array}$} & Primer Ciclo & 89 & 104.02 & & & \\
\hline & Tercer Ciclo & 78 & 117.82 & 2.432 & 2 & .296 \\
\hline & Quinto Ciclo & 50 & 104.11 & & & \\
\hline \multirow{3}{*}{$\begin{array}{l}\text { Dimensión } \\
\text { social }\end{array}$} & Primer Ciclo & 89 & 113.78 & & & \\
\hline & Tercer Ciclo & 78 & 105.41 & .898 & 2 & .638 \\
\hline & Quinto Ciclo & 50 & 106.10 & & & \\
\hline \multirow{3}{*}{$\begin{array}{l}\text { Dimensión } \\
\text { emocional }\end{array}$} & Primer Ciclo & 89 & 105.65 & & & \\
\hline & Tercer Ciclo & 78 & 109.03 & .707 & 2 & .702 \\
\hline & Quinto Ciclo & 50 & 114.93 & & & \\
\hline \multirow{3}{*}{$\begin{array}{l}\text { Dimensión } \\
\text { familiar }\end{array}$} & Primer Ciclo & 89 & 104.64 & & & \\
\hline & Tercer Ciclo & 78 & 112.17 & .736 & 2 & .692 \\
\hline & Quinto Ciclo & 50 & 111.81 & & & \\
\hline
\end{tabular}

* Diferencias significativas al nivel 0.05 (bilateral).

De acuerdo a la tabla 25 se aplicó la prueba no paramétrico Kruskal-Wallis, con el fin de comparar las dimensiones académico, social, emocional y familiar según ciclo de estudios. En todos los casos no se encontraron diferencias estadísticamente significativas.

Tabla 26. Prueba de Anova de un factor para estimar si existen diferencias en los valores interpersonales según ciclo de estudios

\begin{tabular}{ccccccc}
\hline Dimensiones & Grupo & $\begin{array}{c}\text { Suma de } \\
\text { cuadrados }\end{array}$ & gl & $\begin{array}{c}\text { Media } \\
\text { cuadrática }\end{array}$ & F & Sig. \\
\hline \multirow{2}{*}{ Independencia } & Inter-grupos & 173.337 & 2 & & & \\
& Intra-grupos & 5425.916 & 214 & 86.669 & 3.418 & $.035\left(^{*}\right)$ \\
& Total & 5599.253 & 216 & & & \\
Soporte & Inter-grupos & 35.370 & 2 & & & \\
& Intra-grupos & 3823.302 & 214 & 17.685 & .990 & .373 \\
& Total & 3858.673 & 216 & & & \\
\hline
\end{tabular}


VALORES INTERPERSONALES Y AUTOCONCEPTO EN ESTUDIANTES UNIVERSITARIOS DE LA CARRERA DE INGENIERIA AMBIENTAL...

\begin{tabular}{ccccccc}
\hline & Inter-grupos & 138.947 & 2 & & & \\
Benevolencia & Intra-grupos & 5992.435 & 214 & 69.474 & 2.481 & .086 \\
& Total & 6131.382 & 216 & & & \\
& Inter-grupos & 52.624 & 2 & & .393 \\
Conformidad & Intra-grupos & 6000.067 & 214 & 26.312 & .938 & .39 \\
& Total & 6052.691 & 216 & & & \\
& Inter-grupos & 110.828 & 2 & & & \\
\hline \multirow{2}{*}{ Liderazgo } & Intra-grupos & 5714.518 & 214 & 55.414 & 2.075 & \\
& Total & 5825.346 & 216 & & & \\
\hline \multirow{2}{*}{ Reconocimiento } & Intra-grupos & 3268.863 & 214 & 1.988 & .130 & \\
& Total & 3272.839 & 216 & & & \\
\hline
\end{tabular}

* Diferencias significativas al nivel 0.05 (bilateral).

Se aprecia en la tabla 26 las comparaciones según ciclo de estudios de los valores interpersonales a través de la prueba Anova de un factor. Solo se hallaron diferencias significativas $(\mathrm{p}<0.05)$ en el valor independencia.

Tabla 27. Comparaciones múltiples para estimar si existen diferencias en el valor independencia según ciclo de estudios

\begin{tabular}{|c|c|c|c|c|c|c|c|}
\hline & \multirow{2}{*}{$\begin{array}{c}\text { (I) } \\
\text { Ciclo de } \\
\text { estudio }\end{array}$} & \multirow{2}{*}{$\begin{array}{c}(\mathrm{J}) \\
\text { Ciclo de } \\
\text { estudio }\end{array}$} & \multirow{2}{*}{$\begin{array}{l}\text { Diferencia } \\
\text { de medias } \\
\quad(\mathrm{I}-\mathrm{J})\end{array}$} & \multirow{2}{*}{$\begin{array}{l}\text { Error } \\
\text { típico }\end{array}$} & \multirow{2}{*}{ Sig. } & \multicolumn{2}{|c|}{$\begin{array}{c}\text { Intervalo de } \\
\text { confianza al } 95 \%\end{array}$} \\
\hline & & & & & & $\begin{array}{l}\text { Límite } \\
\text { superior }\end{array}$ & $\begin{array}{l}\text { Límite } \\
\text { inferior }\end{array}$ \\
\hline \multirow{6}{*}{$\begin{array}{l}\text { HSD } \\
\text { de } \\
\text { Tukey }\end{array}$} & \multirow{2}{*}{ Primer ciclo } & Tercer ciclo & 1.636 & .781 & .093 & -.21 & 3.48 \\
\hline & & Quinto ciclo & -.506 & .890 & .837 & -2.61 & 1.59 \\
\hline & \multirow{2}{*}{ Tercer ciclo } & Primer ciclo & -1.636 & .781 & .093 & -3.48 & .21 \\
\hline & & Quinto ciclo & -2.142 & .912 & .052 & -4.30 & .01 \\
\hline & \multirow{2}{*}{ Quinto ciclo } & Primer ciclo & .506 & .890 & .837 & -1.59 & 2.61 \\
\hline & & Tercer ciclo & 2.142 & .912 & .052 & -.01 & 4.30 \\
\hline
\end{tabular}


Al encontrarse diferencias significativas en el valor independencia se procedió a identificar en qué ciclos de estudios existían diferencias significativas, para ello se aplicó la prueba post-hoc Tukey. Se obtiene en la tabla 28 que no existen diferencias significativas.

\section{DISCUSIÓN}

Los resultados obtenidos en la presente investigación acerca de los valores interpersonales de los estudiantes de Ingeniería Ambiental y Administración nos muestran que el $42.9 \%$ se encuentra en el nivel bajo en la dimensión independencia, lo que nos indicaría que existe la idea de actuar teniendo en cuenta el criterio de los demás y de no tomar decisiones de manera individual. En la dimensión de soporte, el $42.4 \%$ y el $41.0 \%$ de la muestra se encuentran en un nivel medio y alto respectivamente. El nivel alto significa que este porcentaje de estudiantes sienten la necesidad de ser tratados con comprensión, amabilidad y consideración, además de recibir protección de parte de los demás. García (2010) igualmente encontró en una muestra de estudiantes de una universidad privada, en la que se incluía estudiantes de Administración e Ingeniería, un nivel promedio en soporte. En tanto que el $43.8 \%$ de estudiantes se encuentran en un nivel bajo en la dimensión de benevolencia, evidenciando una disposición negativa para ayudar a los demás y de no mostrarse generoso con ellos. En relación con la dimensión conformidad, el $43.8 \%$ se ubica en un nivel alto, esto significa que podemos encontrar, en un porcentaje considerable, estudiantes dispuestos a hacer lo que socialmente es correcto siguiendo estrictamente las normas, además de hacer lo que es aceptado e idóneo. En la dimensión de liderazgo, el $34.6 \%$ y el 39.2\% de los estudiantes se ubican en el nivel promedio y alto respectivamente. Estos porcentajes indicarían que existe un porcentaje mayor de estudiantes de asumir actitudes de mando y estar a cargo de otras personas teniendo autoridad sobre ellas. Por último, en la dimensión de reconocimiento, encontramos en un nivel alto al $48.4 \%$ de los estudiantes, lo que nos mostraría en ellos una fuerte necesidad de ser admirado, considerado como una persona importante y de conseguir el reconocimiento de los demás.

Analizando la variable autoconcepto, observamos que una mayor proporción (57.6\%) de los estudiantes se ubican en un nivel medio. Similar resultado encontró Sandoval (2009) en su estudio con estudiantes universitarios, donde el 53.8\% se encuentra en un nivel promedio y en igual nivel sus componentes evaluados con el cuestionario de autoconcepto (AF5). Al analizar los resultados de las dimensiones del autoconcepto, apreciamos que en el autoconcepto académico nos muestran que el $37.3 \%$ se ubica en un nivel bajo y que el $37.8 \%$, en un nivel medio. En el autoconcepto social predomina el nivel promedio, en donde se ubican el $65.0 \%$ de los estudiantes. Igualmente sucede en el autoconcepto emocional y el autoconcepto familiar, donde el $55.3 \%$ y el $52.5 \%$ respectivamente se ubican en un nivel medio. En el autoconcepto físico, el $43.8 \%$ de los estudiantes persisten en un nivel medio. 
El análisis correlativo de los resultados nos muestra que el autoconcepto se relaciona con dos valores interpersonales que son soporte y liderazgo. Así, con el valor soporte existe una relación inversa, media y muy significativa. Esto nos indicaría que a un buen nivel de autoconcepto es menor la necesidad de ser tratado con comprensión y apoyo de parte de los demás. Se entiende, asimismo, que un buen autoconcepto se ha constituido a base de un trato digno, respetuoso (Blossom, 2004) y a una serie de creencias que fueron aprendidas por los adolescentes $\mathrm{y}$, por tanto, aquella necesidad de un buen trato va disminuyendo cuanto más se tiene fortalecido el autoconcepto. El hecho de tener un autoconcepto en un nivel aceptable revelaría que son personas que pueden desarrollarse eficazmente en diversas ámbitos de la vida (García y Román, 2003).

La relación mostrada entre el autoconcepto y el valor de liderazgo es directa, media y muy significativa. El conocimiento de las capacidades y cualidades y la percepción de ellos como parte de la identidad personal son aspectos con el cual se caracteriza el líder, de tal manera que un buen líder tiene un buen autoconcepto (Daft, 2007). Identificar esta relación, autoconcepto y liderazgo, en estudiantes de carreras profesionales vinculadas a tareas que conllevan liderar actividades y grupos de personas es significativo en la medida que garantizará el éxito de estas personas en las labores que emprenderán en su trabajo diario.

$\mathrm{Al}$ analizar las relaciones entre los valores interpersonales y las dimensiones del autoconcepto, encontramos que el autoconcepto académico está relacionado muy significativamente con los valores soporte, conformidad y liderazgo. Con el valor soporte se da una relación inversa, implicando que, ante una buena percepción del desempeño académico y rol como estudiante, es menor la búsqueda de apoyo o un trato comprensivo de los demás. Por otro lado, es entendible que la relación sea directa con los valores de conformidad y liderazgo, pues tener conocimiento de la calidad del desempeño como estudiante e ir alcanzando mayor éxito como tal es coherente con la necesidad de actuar conforme a las normas y lo que está aceptado socialmente, además de hacerlo de manera idónea. De igual forma es directa la relación con el valor de liderazgo, ya que al percibirse como buen estudiante es bueno también el sentimiento de sentirse en la capacidad de ejercer el cargo de dirigir y tener autoridad sobre otras personas.

En cuanto al autoconcepto social, la relación es muy significativa con el valor interpersonal de conformidad. Poseer un adecuado conocimiento de las actitudes y del desempeño social, es decir ser amigable, mantener y conservar la amistad e incluso incrementar la red social, implicaría mostrarse como una persona respetuosa de las normas, hacer lo que socialmente es correcto y no mostrarse agresiva o abusar de los demás (Fuentes, García, Gracia y Lila, 2011). Al respecto, García y Musitu (2001, pág. 19) señalan que el autoconcepto social correlaciona positivamente con el ajuste y bienestar psicosocial, con la aceptación y estima de compañeros y con la conducta prosocial.

Otra correlación observada es la que se da entre el autoconcepto emocional y los valores de independencia, soporte, benevolencia, liderazgo. El autoconcepto 
emocional se refiere a la percepción que tienen las personas de su estado emocional y de las respuestas a situaciones específicas experimentadas en su vida diaria. La correlación positiva con el valor independencia indicaría que, al percibir sus emociones y el control que puede ejercer sobre ellas y de las situaciones que enfrenta, se asocia con la sensación de poder actuar con la necesaria libertad para tomar decisiones y responder con criterio propio. La misma relación positiva se da con el valor de liderazgo. De ello se puede deducir que, en cuanto mejor sea la percepción de un adecuado manejo de las emociones y de las situaciones, se estará en mejores condiciones de asumir la dirección y el poder sobre los demás.

Las correlaciones negativas entre la dimensión emocional del autoconcepto y los valores de soporte y benevolencia nos estarían señalando que en los estudiantes no existe la absoluta necesidad de tener un trato amable y comprensivo de parte de los demás o de mostrar por las personas un sentimiento benevolente si se tiene una adecuado conocimiento y manejo de las propias emociones y de las situaciones en las que se pueden encontrar los demás, los cuales buscan motivar actitudes de generosidad hacia ellos. Pareja (2004) encontró resultados similares con estudiantes de quinto de secundaria, donde señala que un mayor desarrollo de la inteligencia emocional disminuye la necesidad de ser tratado con comprensión, amabilidad y consideración. Sin embargo, contrario a nuestro hallazgo, Pareja señala que el valor de benevolencia se correlaciona positivamente con la inteligencia emocional, es decir, que a un mayor desarrollo de la IE, existe una mayor actitud de amor al prójimo y de ayudar a los necesitados. La dimensión familiar del autoconcepto, de acuerdo a la tabla 16, no muestra correlaciones significativas con ninguno de los valores interpersonales. Hernández (1998) encontró que en el único caso donde uno de los aspectos del autoconcepto si tenía una correlación era entre el liderazgo como valor y los sentimientos como miembros de una familia. La correlación apreciada se podría explicar teniendo en cuenta que eran estudiantes universitarios que por algún motivo asistían a al servicio psicológico de la universidad donde estudiaban y que posiblemente veían a la familia como una fuente de apoyo para resolver sus problemas personales.

En los resultados de la tabla 17 encontramos que la dimensión físico del autoconcepto mantiene una correlación negativa pero muy significativa con el valor de soporte y una correlación positiva y significativa con el valor de conformidad. El autoconcepto físico se refiere a la percepción que tienen las personas de su aspecto y condición física (García y Musitu, 2001). Así, de acuerdo a lo hallado, se puede decir que el tener una percepción agradable del aspecto físico, el conocimiento de las habilidades y considerarse bueno para alguna actividad física correlaciona negativamente con la necesidad de ser tratado comprensivamente por las otras personas. Por otro lado, la correlación directa con el valor de conformidad se puede interpretar señalando que, al tener una buena percepción y creencia de lo que puede hacer físicamente y tener un atractivo físico, entonces, se buscará hacer de la mejor forma posible lo que los demás consideran que es correcto o idóneo. En este sentido, García y Musitu (2001) señalan, haciendo referencia a otros autores, que el autoconcepto físico correlaciona positivamente con la percepción de salud, 
con la percepción de bienestar, con el rendimiento deportivo, la motivación de logro y con la integración social (pág. 20).

El análisis comparativo de los resultados a nivel de los promedios y del grado de significancia, tanto de los estudiantes de sexo masculino y femenino en relación con la variable autoconcepto y sus dimensiones físico, académico, social, emocional y familiar, nos ha permitido observar que existen diferencias altamente significativas en la dimensión físico y la dimensión emocional a favor de los estudiantes de sexo masculino. Asimismo se ha encontrado, aunque las diferencias estadísticas no son significativas, que los estudiantes de sexo femenino obtienen mayor promedio en las dimensiones académico, social y familiar. Pero en el autoconcepto general, la media favorece a los estudiantes de sexo masculino.

En relación con los valores interpersonales, analizando las diferencias de los promedios, según el sexo encontramos que existen diferencias estadísticas significativas en el valor de liderazgo a favor de las mujeres. Pareja (2004) obtuvo resultados contrarios, los que indican que los estudiantes varones de quinto de secundaria difieren significativamente de las mujeres en el valor de liderazgo, pues ellos tienen una fuerte necesidad de ser respetados, ser considerados importantes, de tomar decisiones y ejercer autoridad sobre los demás. Sin embargo, sostiene que los resultados analizados no llevan a establecer definitivamente dicha conclusión, pues en la realidad las mujeres van logrando alcanzar posiciones de liderazgo en la sociedad, por ejemplo ocupando puestos importantes en la administración pública.

En los valores de independencia, soporte y conformidad, los estudiantes varones tienen promedios a su favor, mientras que en los valores de benevolencia y reconocimiento las mujeres tienen un promedio por encima del de los varones. Pareja (2004) encontró resultados similares señalando que las mujeres adolescentes del quinto año de secundaria se diferencian de los varones en los valores de benevolencia, pues ellas manifiestan una actitud positiva de amor al prójimo y de ayudar a los que más necesitan. Por otro lado, los resultados difieren a los hallados en nuestra investigación en el valor de reconocimiento, donde la media favorece a los varones y la media del valor conformidad está a favor de las mujeres. Por su parte Camacho (2002) encontró el predominio del valor de soporte, independencia y benevolencia en estudiantes del sexo femenino de cuarto y quinto año de secundaria de la ciudad de Lima y el predominio de los valores de conformidad y liderazgo en los estudiantes de sexo masculino. Observó además que para el caso del valor de reconocimiento no existen diferencias significativas según el sexo.

En los resultados mostrados en la tabla 21 encontramos datos acerca del autoconcepto general y de la dimensión físico en función de las carreras profesionales. Las medias señalan que favorecen a los estudiantes de la carrera de Administración de Empresas. En la tabla 22, las medias de las dimensiones académico, social y familiar son mayores en los estudiantes de la carrera de Administración de Empresas. Solo en la dimensión emocional, la media es mayor en los estudiantes de Ingeniería Ambiental. En ninguna de las dimensiones se halló diferencias estadísticas significativas. Quistgaard (1996) halló que el nivel de autoconcepto es 
más alto en los estudiantes de Ingeniería y Ciencias en relación con lo obtenido por los estudiantes de Administración. Afirma también que el autoconcepto varía según las carreras profesionales, de tal modo que en algunas carreras puede ser mejor que en otras.

La tabla 23 contiene los datos de la media de los valores interpersonales de acuerdo a las carreras profesionales. En el valor de independencia y benevolencia, los estudiantes de Ingeniería Ambiental tienen diferencias a su favor frente a los estudiantes de Administración de Empresas, pero que no son estadísticamente significativas. De igual manera sucede con las medias de los valores soporte, conformidad, liderazgo y reconocimiento, las cuales favorecen a los estudiantes de Administración de Empresas, pero, igualmente, no son estadísticamente significativas. Quesquen (1997) en su investigación encontró que los valores de mayor predominancia en los estudiantes de la carrera de Ingeniería, Medicina, Educación y Derecho de la UNMSM son los valores de reconocimiento y conformidad, pero que en términos generales no se apreciaban diferencias significativas en los valores interpersonales. Fierro (1996), por su lado, afirma que en los estudiantes de Ingeniería Industrial y de Sistemas de la Universidad de Ingeniería, no existen diferencias significativas en los valores interpersonales según el rendimiento académico de dichos alumnos.

Al comparar los datos de los estudiantes del I, III y V ciclo, que corresponden al autoconcepto general así como a sus dimensiones físico, académico, social, emocional y familiar, observamos que no muestran diferencias significativas indicando que son grupos homogéneos. Similar resultado encontró Quistgaard (1996), al plantear la hipótesis para comprobar si los años de estudios afecta el autoconcepto de los estudiantes universitarios del primero al quinto año de seis carreras diferentes (Educación, Ciencias, Humanidades - CC. SS., Ingeniería y Arquitectura, Medicina - Ciencias de la Salud, Ciencias Contables y Administración), procedentes de dos universidades estatales y dos privadas, concluye que los años de estudios no influyen en las diferencias.

En relación con los resultados de los valores interpersonales, hallamos que el valor independencia difiere significativamente en los grupos. Sin embargo, al querer identificar en qué ciclos existen las diferencias encontramos que los grupos de los tres ciclos, según la prueba HSD de Tukey, no muestran diferencias estadísticas significativas, lo que indica que son grupos homogéneos en cuanto a la forma de expresar el valor de independencia. García (2010) en su investigación señala que el eje transversal de ética y valores ha influido en el desarrollo de los valores interpersonales de conformidad y reconocimiento y que existen diferencias entre los alumnos del I ciclo y VIII ciclo de diferentes escuelas profesionales. Asimismo, manifiesta también que los estudiantes de los ciclos señalados no presentan diferencias significativas en los valores de soporte, independencia, benevolencia y liderazgo. Estos resultados apoyarían en cierto modo que los ciclos académicos no tienen influencia decisiva en las expresión de dichos valores en los estudiantes universitarios. 
VALORES INTERPERSONALES Y AUTOCONCEPTO EN ESTUDIANTES UNIVERSITARIOS DE LA CARRERA DE INGENIERIAA AMBIENTAL...

\section{CONCLUSIONES}

1. Los valores interpersonales se presentan en diferentes niveles en tanto que el autoconcepto y sus dimensiones se encuentran en un nivel medio en la mayoría de los estudiantes.

2. Los valores interpersonales de soporte y conformidad se encuentran correlacionados con el autoconcepto general. Asimismo, considerando las dimensiones del autoconcepto, se puede señalar que el valor de soporte está relacionado con la dimensión del autoconcepto académico, emocional y físico; el valor de conformidad está relacionado con las dimensiones del autoconcepto académico, social y físico. El liderazgo se relaciona con el autoconcepto académico y emocional. El valor de independencia y benevolencia se relacionan con las dimensiones del autoconcepto emocional y social, respectivamente. Por último, ninguno de los valores interpersonales se relacionan con el autoconcepto familiar.

3. Existen diferencias estadísticamente significativas en el valor interpersonal de liderazgo, según el sexo, a favor de las mujeres.

4. Según el sexo, existen diferencias altamente significativas en el autoconcepto físico y emocional a favor de los varones, mientras que se aprecian diferencias significativas en el valor interpersonal de liderazgo a favor de las mujeres.

5. Existen diferencias estadísticamente significativas en el valor interpersonal de independencia según el ciclo de estudios, mientras que no se observan diferencias estadísticamente significativas en los valores interpersonales según la carrera profesional.

6. No existen diferencias estadísticamente significativas en el autoconcepto de los estudiantes, varones y mujeres, según el ciclo académico y la carrera profesional que cursan.

\section{REFERENCIAS BIBLIOGRÁFICAS}

Aguilar, S. (2002). Estudio comparativo de los valores interpersonales en alumnos con alta y baja autoestima de los tres primeros ciclos de la Escuela de Psicología de la Universidad Señor de Sipán en la ciudad de Chiclayo. Tesis para obtener el título de licenciado en Psicología. Universidad César Vallejo.

Álvaro, J. et al (2012). Actitudes de los estudiantes españoles ante la ampliación de los derechos sociales de hijos de inmigrantes: Un análisis de los valores como discurso legitimador. Persona. $\mathrm{N}^{\circ} 15$, enero - diciembre, pp. 99 - 114.

Berninzón, E. (1996). Los valores interpersonales en alumnos de psicología dela Universidad de San Martín de Porres. Tesis para optar el grado académico de Maestría en Piscología. USMP. Lima - Perú.

Blossom, M. (2004). Entendiendo a los adolescentes. México: Selector, S. A. 
Camacho, S. (2000). El análisis transaccional y la mejora de las relaciones interpersonales. Revista Comunicar N 14, pp. 133 - 136. Recuperado el 25 de julio del 2013 de http:// www.redalyc.org/pdf/158/15801417.pdf

Camacho, L. (2002). Relación entre la percepción del tipo de familia y los valores interpersonales en adolescentes de cuarto y quinto grados de secundaria de LimaCercado. Tesis de Magister en Psicología con mención en Psicología Educativa. UNMSM. Lima - Perú.

Carmona, B. y León, M. (2005). Análisis correlacional entre las inteligencias múltiples interpersonales e intrapersonales y valores interpersonales, en estudiantes de I ciclo de la carrera de Psicología de la Universidad César Vallejo de la ciudad de Trujillo. Tesis de Licenciatura. Universidad César Vallejo. Trujillo - Perú.

Daft, R. (2007). La experiencia del liderazgo. Madrid: Editorial Thomson Paraninfo.

Fierro, E. (1996). Relación entre los valores interpersonales y el rendimiento académico en alumnos de ingeniería industrial y de sistemas de la U.N.I. Tesis para el título licenciada en Psicología. UIGV. Lima - Perú.

Fuentes, M., García, J., Gracia, E y Lila, M. (2011). Autoconcepto y ajuste psicosocial en la adolescencia. Universidad de Valencia. En Psicothema. Vol. 23, No 1, pp. 7-12. Recuperado el 15 de marzo del 2013 de http://www.psicothema.com/pdf/3842.pdf

García, L. (2005). Sistema valorativo de estudiantes de pregrado y preuniversitarios de la Pontificia Universidad Católica del Perú. Tesis para licenciatura en Psicología con Mención en Psicología Social.

García, T. (2010). Valores interpersonales en estudiantes del I y VIII ciclo académico de una universidad privada. Revista Temática Psicológica. Universidad Femenina del Sagrado Corazón. UNIFE. Enero - Diciembre, Vol. 6, N 1, pp. 21- 33.

García, F. y Musitu, G. (2001). Autoconcepto Forma 5. Madrid: TEA Ediciones S. A.

García, J. y Román, J. M. (2003). Educación familiar y autoconcepto en niños pequeños. Madrid: Ediciones Pirámide.

Gordon, L. (1995). Cuestionario de valores interpersonales. Madrid: TEA Ediciones S. A.

Hernández, Y. (1998). Relación de Liderazgo y Autoconcepto en un grupo de estudiantes universitarios. Tesis para optar el título profesional de Licenciado en Psicología. USMP. Lima - Perú.

Molero, F. (2003). Psicología social de los valores humanos. Desarrollos teóricos, metodológicos y aplicados. Revista de Psicología del Trabajo y de las Organizaciones. Vol. 19, $\mathrm{N}^{\circ}$ 2, pp. 215 - 218. Recuperado el 14 de agosto del 2013 de http://www. redalyc.org/pdf/2313/231318052006.pdf

Navarro, V. (2009). "Valores interpersonales y juicio moral en estudiantes de dos universidades particulares de Lima Metropolitana”. Tesis para optar el grado de Magister en Psicología con mención en Psicología Educativa. UNMSM. Lima - Perú. 
VALORES INTERPERSONALES Y AUTOCONCEPTO EN ESTUDIANTES UNIVERSITARIOS DE LA CARRERA DE INGENIERIA AMBIENTAL...

Pareja, A. (2004). La Inteligencia emocional y su relación con los valores interpersonales en estudiantes del 5to. año de educación secundaria. Tesis para optar el grado de Magister en Psicología con mención en Psicología Educativa. UNMSM. Lima - Perú.

Pérez, N. y Navarro, I. (2011). Psicología del desarrollo humano: del nacimiento a la vejez. España: Editorial Club Universitario.

Portilla, L. (2011). Calidad de vida y Autoconcepto en pacientes con Diabetes Mellitus con y sin adherencia al tratamiento. Tesis para optar el título de Psicólogo. UNMSM. Lima - Perú.

Quesquen, F de M. (1997). Valores interpersonales prevalentes en los estudiantes del I ciclo de las facultades de ingeniería, medicina, educación y derecho en la U.N.M.S.M. Tesis para optar el grado de Maestro en Educación. USMP. Lima - Perú.

Quistgaard, J. (1996). Los niveles de autoconcepto y conducta tipo A y B en estudiantes universitarios de Lima Metropolitana. Tesis para optar el grado académico de Maestría en Psicología. UIGV. Lima - Perú.

Restrepo, J., Martínez, G., Soto, J., Martínez, F. y Baena, B. (2009). Valores personales e interpersonales en adolescentes y adultos de la ciudad de Medellín y el área metropolitana. Diversitas: Perspectivas en Psicología. Vol. 5. $\mathrm{N}^{\circ} 1$, pp. $125-$ 139. Recuperado el 27 de julio del 2013 de http://www.scielo.org.co/pdf/dpp/v5n1/ v5n1a11.pdf

Reyes, Y. (2003). Relación entre el rendimiento académico, la ansiedad ante los exámenes, los rasgos de personalidad, el autoconcepto y la asertividad en estudiantes del primer año de psicología de la UNMSM. Tesis para optar el título de Psicólogo. UNMSM. Lima - Perú. En http://sisbib.unmsm.edu.pe/bibvirtualdata/tesis/salud/reyes_t_y/t_ completo.pdf

Sandoval, Y. (2009). Autoconcepto y factores de protección asociados al consumo de alcohol y tabaco en universitarios. Tesis para optar el grado de Magister. UNFV. Lima - Perú. 